\title{
Malformação de Chiari: Relato de caso
}

\author{
Chiari malformation: Case report \\ Malformación de Chiari: Reporte de caso
}

Recebido: 17/05/2021 | Revisado: 27/05/2021 | Aceito: 28/05/2021 | Publicado: 12/06/2021

Letícia Góes Pereira

ORCID: https://orcid.org/0000-0002-0331-190X Faculdade Morgana Potrich, Brasil E-mail:leticiagoes98@hotmail.com

Açucena de Oliveira Borges

ORCID: https://orcid.org/0000-0003-1781-0870

Faculdade Morgana Potrich, Brasil

E-mail: assucena-o-b-@ hotmail.com

Gustavo Lúcio Monteiro de França

ORCID: https://orcid.org/0000-0002-7789-8432

Faculdade Morgana Potrich, Brasil

E-mail: gstvlucio@gmail.com

Artur Cunha Vasconcelos

ORCID: https://orcid.org/0000-0003-0633-9569

Faculdade Morgana Potrich, Brasil

E-mail: arturcunha@fampfaculdade.com.br

\begin{abstract}
Resumo
As malformações de Chiari (MC) constituem anormalidades na base do cérebro que envolvem estruturas como cerebelo, o tronco encefálico e a junção crânio cervical. A etiopatogenia dessa doença ainda não é bem esclarecida, acredita-se que é uma alteração congênita, devido a malformações na embriogênese. O presente relato tem como objetivo identificar a gravidade da doença em pacientes diagnosticados com Malformação de Chiari tipo I, a importância do diagnóstico precoce e a intervenção cirúrgica para redução de sequelas. A metodologia utilizada neste estudo de caso foi realizada de forma qualitativa e descritiva, por coleta direta dos dados da paciente por meio do acesso aos prontuários e exames por ela prestados. Os resultados encontrados foram que a sintomatologia pode variar desde cefaleias occipitais até déficits motores e sensoriais. O diagnóstico geralmente ocorre na idade adulta, por meio de ressonância magnética, sendo o tratamento apenas cirúrgico. As imagens de ressonância magnética revelam invaginação vertebrobasilar, clivus curto congênito e herniação das tonsilas cerebelares que obstruíram o canal vertebral fazendo compressão bulbomedular. A abordagem cirúrgica foi realizada pela retirada de uma parte inferior do osso occipital e arco posterior de $\mathrm{C} 1$. O caso teve evolução favorável e estável, porém o paciente ainda apresenta sequelas em decorrência da gravidade do quadro clínico. Conclui-se assim, que se trata de uma doença rara e de alta complexidade devido a especificidade da intervenção cirúrgica, sendo o diagnóstico precoce um importante aliado na redução de sequelas.
\end{abstract}

Palavras-chave: Anomalia; Invaginação vertebrobasilar; Herniação; Tonsilas cerebelares; Compressão bulbomedular.

\begin{abstract}
Chiari (MC) malformations are abnormalities at the base of the brain that involve structures such as the cerebellum, the brainstem and the cervical cranial junction. The etiopathogenesis of this disease is still unclear, it is believed that it is a congenital alteration, due to malformations in embryogenesis. This report aims to identify the severity of the disease in patients diagnosed with Chiari Malformation type I, the importance of early diagnosis and surgical intervention to reduce sequelae. The methodology used in this case study was carried out in a qualitative and descriptive manner, through direct collection of patient data through access to medical records and exams provided by her. The results found were that the symptoms can vary from occipital headaches to motor and sensory deficits. The diagnosis usually occurs in adulthood, by means of magnetic resonance imaging, and the treatment is only surgical. Magnetic resonance images reveal vertebrobasilar invagination, congenital short clivus and herniation of the cerebellar tonsils that obstructed the vertebral canal with bulbomedular compression. The surgical approach was performed by removing a lower part of the occipital bone and posterior arch of $\mathrm{C} 1$. The case had a favorable and stable evolution, but the patient still has sequelae due to the severity of the clinical condition. It is concluded, therefore, that it is a rare and highly complex disease due to the specificity of the surgical intervention, with early diagnosis being an important ally in the reduction of sequelae. Keywords: Anomaly; Vertebrobasilar invagination; Herniation; Cerebellar tonsils; Bulbomedular compression.
\end{abstract}




\section{Resumen}

Las malformaciones de Chiari (MC) son anomalías en la base del cerebro que involucran estructuras como el cerebelo, el tronco encefálico y la unión craneal cervical. La etiopatogenia de esta enfermedad aún no está clara, se cree que es una alteración congénita, debido a malformaciones en la embriogénesis. Este informe tiene como objetivo identificar la gravedad de la enfermedad en pacientes diagnosticados con malformación de Chiari tipo I, la importancia del diagnóstico temprano y la intervención quirúrgica para reducir las secuelas. La metodología utilizada en este estudio de caso se llevó a cabo de manera cualitativa y descriptiva, mediante la recolección directa de datos del paciente mediante el acceso a historias clínicas y exámenes proporcionados por ella. Los resultados encontrados fueron que los síntomas pueden variar desde dolores de cabeza occipitales hasta déficits motores y sensoriales. El diagnóstico suele darse en la edad adulta, mediante resonancia magnética, y el tratamiento es solo quirúrgico. Las imágenes de resonancia magnética revelan invaginación vertebrobasilar, clivus corto congénito y herniación de las amígdalas cerebelosas que obstruían el canal vertebral con compresión bulbomedular. El abordaje quirúrgico se realizó mediante la extirpación de una parte inferior del hueso occipital y arco posterior de C1. El caso tuvo una evolución favorable y estable, pero el paciente aún presenta secuelas por la gravedad del cuadro clínico. Se concluye, por tanto, que se trata de una enfermedad rara y de gran complejidad por la especificidad de la intervención quirúrgica, siendo el diagnóstico precoz un aliado importante en la reducción de secuelas.

Palabras-clave: Anomalía; Invaginación vertebrobasilar; Hernia; Amígdalas cerebelosas; Compresión bulbomedular.

\section{Introdução}

As malformações de Chiari (MC) representam anomalias na base do cérebro que envolvem estruturas como cerebelo, o tronco encefálico e a junção crânio cervical, causando aumento do diâmetro do Forame Magno e adensamento da fossa posterior do crânio (Hoederath et al., 2014; Oliveira et al., 2011; Mascarenhas et al., 2018).

Cleland em 1883 descreveu pela primeira vez o alongamento do IV ventrículo e a herniação das tonsilas cerebelares inferiormente no forame magno. Em 1891 Chiari descreveu três tipos de deformidades do rombencéfalo e anormalidades morfológicas da fossa posterior craniana, já em 1896 escreveu o quarto tipo da malformação (Silva et al., 2003; Araújo et al., 2017). O epônimo malformação de Arnold descrita por Cleland designava herniação cerebelar e o epônimo malformação de Chiari designava malformação no rombencéfalo, assim Schwalbe e Gredig em 1907 propuseram o epônimo Arnold-Chiari para malformação do rombencéfalo, porém na literatura prevalece o epônimo malformação de Chiari (Silva et al., 2003).

As malformações de Chiari podem ser classificadas em quatro tipos, com forme sua morfologia e gravidade dos defeitos anatômicos, diferenciadas por meio de exames de imagens ou autópsia (Hidalgo et al., 2020). O tipo I é o mais comum e é caracterizado pelo deslocamento caudal das tonsilas cerebelares e da parte medial e inferior do lobo posterior do cerebelo pelo canal vertebral, acima de 3 a 5mm a partir do forame magno (Oliveira et al., 2011; Brito et al., 2019). Raramente é visto abaixo de C2 e geralmente é diagnosticada na vida adulta (Vale et al., 2014). A mielomeningocele é ausente e a fossa posterior possui dimensões reduzidas (Magalhães et al., 2019); o tipo II é uma anormalidade do sistema nervoso central, está associado a uma mielodisplasia que é a mielomeningocele e hidrocefalia. As hérnias não são apenas tonsilares, mas também do vérmis, quarto ventrículo, bulbo, hemisférios cerebelares e ponte em direção ao canal vertebral. Morfologicamente, ocorrem estenose aquedutal, hidromelia e displasia cortical (Hoederath et al., 2014). É também, relatada abaixo de C2. Por fim é caracterizada por múltiplas anomalias de fossa posterior, associadas com a herniação (Magalhães et al., 2019; Moro et al., 1999); o tipo III, a mais grave, é uma encefalocele occipital baixa ou cervical alta acarretada pela descida do cerebelo e tronco cerebral pela coluna vertebral (Hoederath et al., 2014; Magalhães et al., 2019; oliveira et al., 2019); o tipo IV atualmente é um termo obsoleto (Arora, 2015; Hidalgo et al., 2020). Antes era caracterizada como uma hipoplasia severa ou aplasia do cerebelo, semelhante a agenesia cerebelar primária, associada à fossa posterior, ambos apresentando na maioria dos casos pequena dimensão, sem herniação (Hoederath et al., 2014; Moro et al., 1999; Araújo et al., 2017).

A patogenia ainda não é bem estabelecida, porém existem algumas evidências em relação a malformação tipo I, ao qual possui um defeito do mesoderma paraxial após o fechamento do tubo neural o que pode levar ao hipodesenvolvimento do basio condrocrânio, resultando em anomalias estruturais da base do crânio. Além de segmentação anormal dos corpos vertebrais 
cervicais, superlotação causada por uma pequena abóbada craniana na fossa posterior e tecido excessivo na fossa posterior ou na abóbada craniana inteira (Hoederath et al., 2014; Souza et al., 2019; Martins et al., 2008).

Os sintomas são consequências da compressão de nervos cranianos, cerebelo, tronco cerebral e medula espinhal. Porém os pacientes podem ser assintomáticos, ter sintomas inespecíficos ou ter déficits neurológicos progressivos e graves (Hale et al., 2016). As sintomatologias usuais são cefaleias, dor no pescoço, déficits motores, atrofia muscular, paralisia craniana inferior, ataxia cerebelar, nistagmo, déficits sensoriais, disfagia, disfonia e comorbidades psiquiátricas, como transtornos de ansiedade e humor (Hoederath et al., 2014).

Os déficits cognitivos podem ser resultantes de lesão direta dos sistemas cerebelares ou do tronco cerebral, ou consequência da ansiedade e depressão que são comuns em paciente com dor crônica (Allen et al., 2014).

No tipo I, os sintomas aparecem entre 30 e 50 anos de idade, mais frequente em mulheres (1,3: 1), acompanhadas de manifestações motoras, sensoriais e autonômicas como cefaleia occipital, atrofia muscular e parestesia das extremidades superiores. Essas se agravam pela flexão, extensão cervical ou tosse, síndrome cerebelar, com incoordenação motora e desequilíbrio (Oliveira et al., 2014; Hidalgo et al., 2020). Uma das condições relatadas em pacientes com Chiari tipo I, em 40$76 \%$ dos casos, é a siringomielia. Caracterizada pela formação de uma ou mais cavidades contendo LCR dentro da medula espinhal, sendo distúrbio mais comum, esta malformação começa na região cervical (Hale et al., 2016).

O diagnóstico de MC-I é dado pela ressonância magnética da cabeça e da coluna cervical que mostra a herniação das tonsilas cerebelares através do forame magno com descensão de $5 \mathrm{~mm}$, bloqueio do LCR e compressão medular, juntamente a sintomas clínicos como a dor de cabeça (Silva et al., 2003; Allen et al., 2014; Hidalgo et al., 2020).

\section{Metodologia}

Este estudo de caso foi realizado de forma qualitativa e descritiva (Pereira et al., 2018), por coleta direta dos dados da paciente por meio do acesso aos prontuários e exames por ela prestados. Os dados foram coletados após o diagnóstico da paciente para malformação de Chiari I juntamente com invaginação vertebrobasilar e clivus curto congênito. Assim, foram analisados os sinais, sintomas, tratamento e consequências pós operatórias da paciente em questão. Este estudo de caso teve aprovação do Comitê de Ética em Pesquisa e também se realizou o Termo de Consentimento Livre e Esclarecido, o mesmo foi assinado pela paciente. Sendo respeitados os princípios éticos da Declaração de Helsinque.

\section{Relato do Caso}

Paciente do sexo feminino, 38 anos de idade, branca, apresenta histórico de distúrbio crônico de equilíbrio, incoordenação e fraqueza nos quatro membros levando a quedas frequentes e quadro de cefaleias intensas em região occipital. Relata que o aparecimento dos primeiros sintomas tenha ocorrido mais ou menos aos 22 anos de idade. Foi devidamente, avaliada, investigada e diagnosticada através de exames de ressonâncias magnéticas crânio-cervicais e caracterizada com síndrome cerebelar grave de compressão do tronco encefálico e medula cervical alta, com nistagmo, disfagia, ataxia e tetraparesia, decorrente de malformação de Chiari I juntamente com invaginação vertebrobasilar e clivus curto congênito.

Ao exame de ressonância magnética do crânio e da região cervical realizado antes da cirurgia (Figura 1) observa-se herniação das tonsilas cerebelares com compressão bulbomedular grave e clivus curto congênito, fazendo a obliteração nessa região, diminuindo o trânsito do líquido cefalorraquidiano, que é a malformação de Chiari I. 
Figura 1. Ressonância magnética em secção sagital das regiões cervical e craniana, evidenciando a herniação das tonsilas cerebelares.

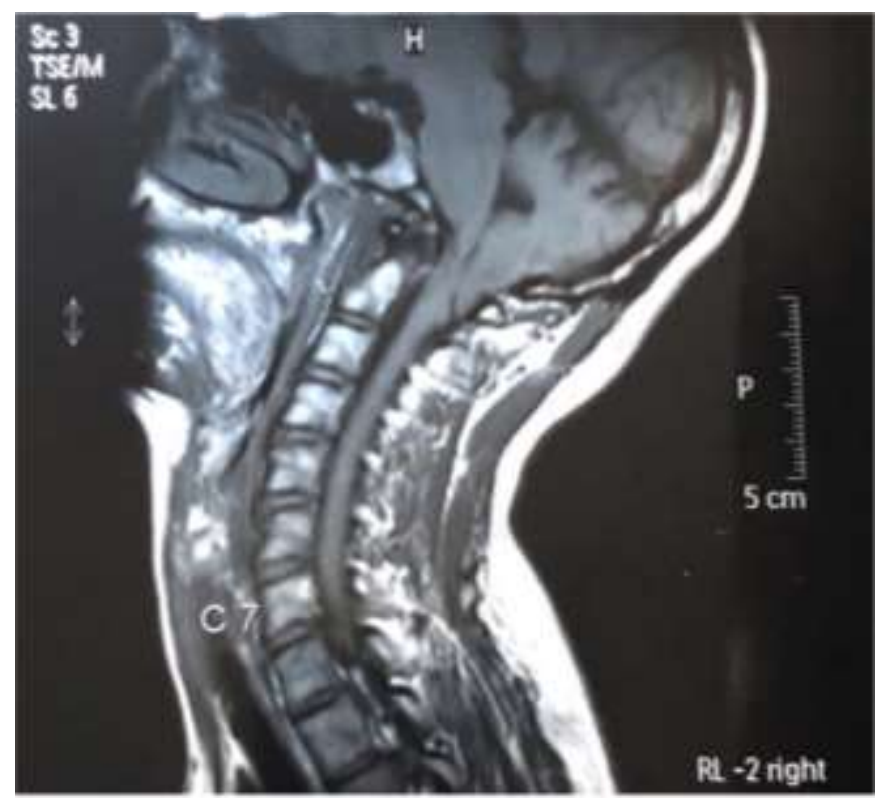

Fonte: Autores.

O tratamento consistiu em um procedimento cirúrgico com objetivo de reduzir essa compressão bulbomedular, liberando a passagem do líquido cefalorraquidiano, retirando-se uma parte inferior do osso occipital e arco posterior de $\mathrm{C} 1$ (primeira vertebra cervical) (Figura 2), após esse procedimento, a coluna vertebral ficou instável e foi necessário a fixação crâniocervical posterior com parafusos desde o osso occipital até a vértebra C4 (Figura 3). 
Research, Society and Development, v. 10, n. 7, e3310716202, 2021

(CC BY 4.0) | ISSN 2525-3409 | DOI: http://dx.doi.org/10.33448/rsd-v10i7.16202

Figura 1. Ressonância magnética após descompressão bulbomedular.

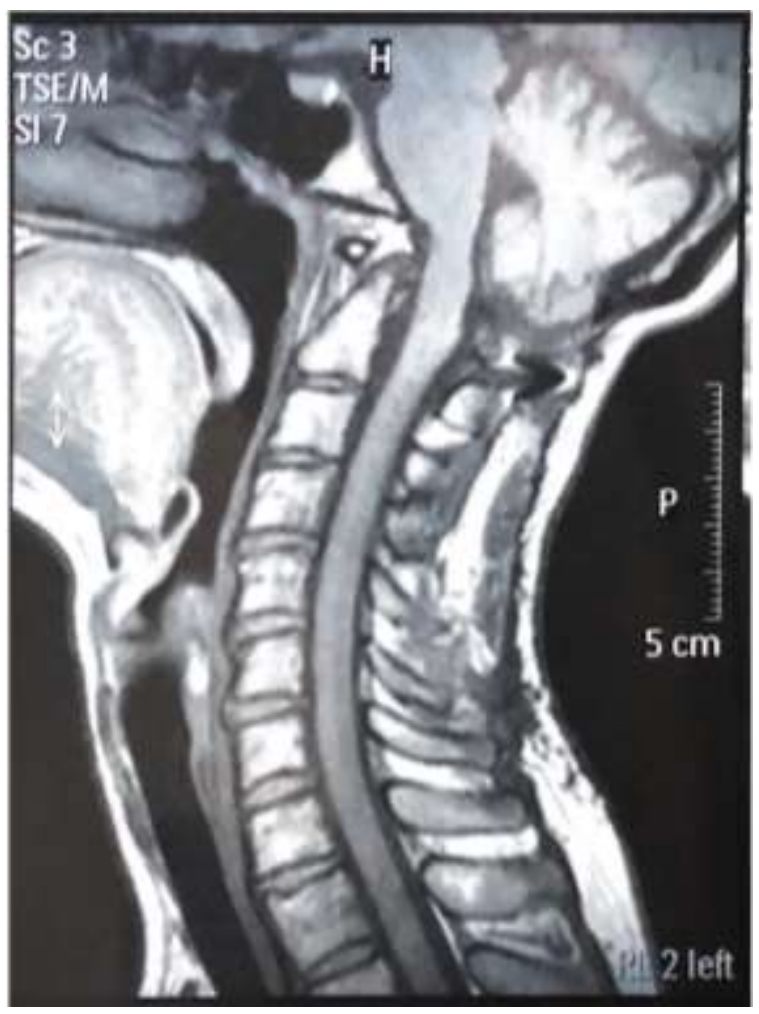

Fonte: Autores.

Figura 3. Raio X da região cervical demonstrando fixação crâniocervical com parafusos até o limite de C4.

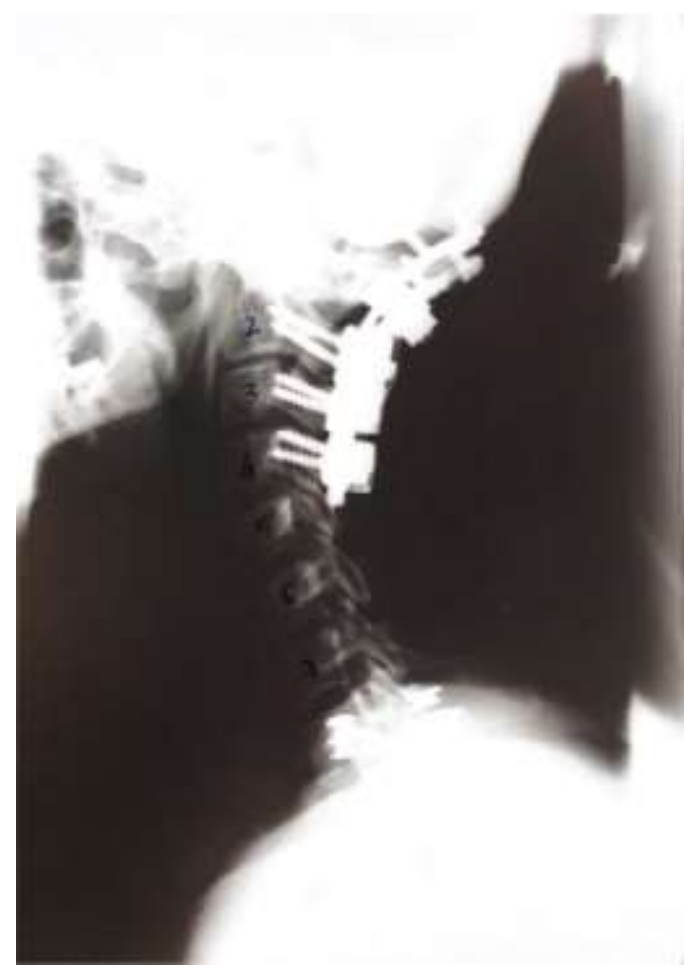

Fonte: Autores. 
Além disso, a operação foi bem sucedida em termos de descompressão e estabilização da invaginação basilar. A paciente apresentou recuperação favorável, com melhora parcial e estabilização do quadro neurológico. Algumas sequelas permanecem evidentes como nistagmo, ataxia e tetraparesia discreta. A deambulação é possível, mas com limitações neurológicas.

\section{Discussão}

A malformação de Chiari I é habitualmente assintomática na infância, porém ao longo dos anos vão surgindo disfunções progressivas. Essas se manifestam com sintomas clínicos diversos, variando conforme o local afetado (medula espinhal cervical ou compressão primária do tronco encefálico ou cerebelo). Caso comprima o cerebelo manifestam-se ataxia e nistagmo. Já quando afetado o tronco encefálico podem surgir cefaleia, cervicalgia e alterações dos nervos cranianos baixos, causando disfunção das suas respectivas atividades motoras e sensitivas. Por outro lado, quando a medula espinhal é afetada o paciente pode manifestar disestesia de tronco e extremidades, paresia de membros superiores, com hipotrofia ou atrofia de musculatura das mãos, espasticidade nos membros inferiores. Assim como perdas sensitivas dissociadas no tronco e membros superiores e incontinência urinária (Magalhães et al., 2019; Oliveira et al., 2019; Araújo et al., 2020; Kular, et al., 2021).

A ressonância magnética e a tomografia computadorizada são os exames de imagens para o diagnóstico e monitorização da malformação de Chiari I, pois revela a herniação das tonsilas cerebelares que se estendem 5 mm ou mais para baixo do forame magno (Souza et al., 2019). E é feito o diagnostico diferencial de outras patologias como esclerose múltipla, siringomielia, desenvolvimento defeituoso do atlas e do áxis, hipotensão cranioespinhal, hipertensão intracraniana idiopática e invaginação basilar (Araújo et al., 2017; Kular, et al., 2021).

O tratamento cirúrgico possibilita a melhora sintomatológica e da qualidade de vida, através da descompressão da fossa posterior do crânio com reestabelecimento de fluxo do líquido cefalorraquidiano adequado ao nível de forame magno. A falta de intervenção cirúrgica pode resultar em agravamento da doença e piora dos sintomas (Moro et al., 1999; Vidal et al., 2015).

Pode ocorrer complicações pós operatórias como infecção da ferida operatória, meningite, paralisia dos nervos cranianos, edema da fossa posterior, hidrocefalia, mutismo cerebelar, vazamento de LCR, formação de pseudomeningocele, meningite asséptica e bacteriana, lesão da artéria vertebral e formação de hematoma epidural, caso se manifestem é possível necessário uma nova intervenção cirúrgica (Lobão et al., 2020; Kular et al., 2021).

\section{Conclusão}

A cirurgia que minimiza a compressão bulbomedular, osteotomia parcial do forame magno do osso occipital e arco posterior da primeira vertebra cervical, libera a passagem do líquido cefalorraquidiano, pode ser o melhor meio de tratamento da malformação de Chiari. Por se tratar de uma doença de alta complexidade, o diagnóstico precoce é fundamental para a redução das sequelas, o que permite a melhora dos sintomas e da qualidade de vida do paciente. Para a melhor compreensão deste quadro, sugere-se a realização de trabalhos com maior espectro amostral.

\section{Referências}

Allen, P. A., Houston, J. R., Pollock, J. W., Buzzelli, C., Li X., Harrington A. K., Martin B. A., Loth F., Lien M. C., Maleki J., \& Luciano M. G. (2014). Efeitos cognitivos gerais e específicos da tarefa na malformação de Chiari tipo I. PLOS ONE. 9(4): 1-11. 10.1371 / journal. pone.0094844.

Araújo, I. A., Amorim, E. F., Santana, L. M. M., Alencar, E. P., Araújo, M. I. A., \& Fernandes, P. E. (2017). Malformação de Arnold-Chiari: uma revisão bibliográfica. Journal of Medicine and Health Promotion, 2(3), 661-669.

Araújo, M. L., Alves Sousa, M. N., \& Araújo Neto, S. A. (2020). Prevalência das malformações associadas à invaginação basilar e suas manifestações clínicas. Revista Brasileira Multidisciplinar, 23(3), 150-164. https://doi.org/10.25061/2527-2675/ReBraM/2020.v23i3.798

Arora, R. (2015). Espectro de imagem das patologias cerebelares: um ensaio pictórico. Jornal polonês de radiologia, 80, 142150. https://doi.org/10.12659/PJR.892878 
Brito, N. P., et al. (2019). Basilar invagination associated with chiari malformation type I: A literature review. Clinics, 74(653), 1-6. https://doi.org/10.6061/clinics/2019/e653.

Hale, O., Alper, D., Avla, K., Handan, S., Canan, Y., \& Can, P. (2016). Prevalência de malformação de chiari tipo I na imagem por ressonância magnética cervical: Um Estudo Retrospectivo. Anatomy. 10(1): 40-45. 10.2399/ana.15.039.

Hidalgo, J. A., Tork, C. A., \& Varacallo, M. Arnold Chiari Malformation. (2020). Arnold Chiari Malformation. StatPearls. 1-18. Available in: https://www.ncbi.nlm.nih.gov/books/NBK431076/.

Hoederath, L., Jellestad, L., Jenewein, J., \& Boettger, S. (2014). Psychotic and major neurocognitive disorder secondary to arnold-chiari type II malformation. Psychiatr Danub. 26(3):291-293.

Kular, S., \& Cascella. M. (2021). Chiari I Malformation. StatPearls, 1-20. Ahttps://www.ncbi.nlm.nih.gov/books/NBK554609/

Lobão, C. A. F., Sousa, U. O., Cabral, D. A. C., \& Campos, F. M. S. (2020). Hemifacial Spasm Associated with Chiari Type I Malformation: Surgical Considerations and Case Report. Arq Bras Neurocir, 39(2), 136-141. DOI https://doi.org/10.1055/s-0040-1708893

Magalhães, M. J. S., Pimenta, B. P. M., Gomes, H. O., Neto, J. V. F., Oliveira, R. V., \& Novais, S. S. (2019). Alterações neuroanatômicas do encéfalo na malformação Arnold-Chiari II. Revista Eletrônica Acervo Saúde. (19):1-8. https://doi.org/10.25248/reas.e710.2019

Martins, H. A. L., Ribas, V. R., \& Valença, M. M. (2008). Cefaleia associada à malformação de Chiari do tipo I. Migrâneas cefaleias.11(4): 238-244.

Mascarenhas, M. L. V. C., Ferreira, A. L. C., Acioli, D. M. N., Oliveira, D. N. \& Lúcio, I. M. L. (2018). Mielomeningocele e sinais de mal formação de arnold chiari II em lactente. GEP NEWS, 1(1), 215-219.

Moro, E. R. P., Teive, H. A. G., Souza, S. M. P., Lambrecht, F., \& Werneck, L. C. (1999). Malformação de Chiari Tipo I. Arq Neuropsiquiatr.57(3-A): 666671.

Oliveira, N. F. C. D., Oliveira, R. C. C. D., \& Paula, J. M. (2011). Manifestações otoneurológicas tardia da malformação de Chiari I. Arq Int Otorrinolaringol.15(3):382-384. DOI: 10.1590/S1809-48722011000300018

Oliveira, T. P., Oliveira, R. S., Silva, E. M. C., \& Pereira, E. D. M. (2019). Qualidade de vida em paciente com Arnold Chiari Tipo II - Relato de Caso. Revista de Patologia do Tocantins. 6(3):17-21.

Pereira, A. S. et al. (2018). Metodologia da pesquisa científica. [e-book]. Santa Maria. Ed. UAB/NTE/UFSM. Available: https://repositorio.ufsm.br/bitstream/handle/1/15824/Lic_Computacao_Metodologia-Pesquisa-Cientifica.pdf?sequence=1. Accessed in: 07 nov. 2019.

Silva, J. A. G., Brito, J. C. F., Taricco, M. A., Arruda, J. A. M., Masini, M., \& Filho, G. S. C. (2003). Malformações Occipitocervicais. Recife: Editora Universitária UFPE.

Souza, I. A., Gomes, M. B., Ramires, G. E., Campos, F. F., \& Baracat, F. I. (2019). Malformação de Chiari I: Relato de Caso. Rev UNINGÁ. 56(2):44-49.

Vale, J. M., Silva, E., Pereira, I. G., Marques, C., Serrano, A. S., \& Torres, A. S. (2014). Malformação de Chiari e síndrome de apneia central do sono: eficácia do tratamento com servoventilação adaptativa. J Bras Pneumol. 40(5):574-578. http://dx.doi.org/10.1590/S1806-37132014000500014

Vidal, C. H. F. (2015). Tratamento cirúrgico da malformação de Chiari tipo I: o papel da abertura do forame de Magendie e manipulação das amígdalas. Arquivos de Neuro-Psiquiatria, 73 (2), 179. https://doi.org/10.1590/0004-282X20140220 